\title{
Are predictive risk scores useful?
}

0 ver the last three decades we have witnessed a dramatic reduction of recurrent cardiovascular events in patients with established cardiovascular disease (CVD). This advance in secondary prevention is due mainly to evolving aggressive medical and interventional therapies. However, risk-factor modification in primary prevention-that is, prevention of the first, often fatal, CVD event-is also thought to be a contributing factor.

Over the same period of time, the Framingham Heart Study investigators and many others have made exceptional contributions in recognizing a number of risk factors or causes that cluster and interact multiplicatively in predicting primary CVD events. Algorithms for the prediction of 10-year CVD events have been developed on the basis of epidemiological evidence; each individual risk factor has been quantified and given a value that adds to the final total predictive risk score, often associated with the generic connotation of high, intermediate or low

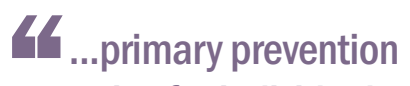
strategies for individual patients should be simple and personal 77 audience of approximately 2,000 cardiologists and internists a simple question: "For evaluation and management of risk factors, the scientific community and some of the guidelines refer to high, intermediate or low risk scores; how many of you have the scoring tables or charts in your offices?" The answer was astonishing and reassured me of my personal position. Only 11 out of the approximately 2,000 audience members raised their hands. To have an impact on primary prevention, we should thus begin by simplifying our approach to the evaluation and management of risk factors.

Support of a more simplistic approach in primary care comes from the INTERHEART investigators who evaluated 30,000 individuals in 52 countries and concluded that each of the abovementioned individual risk factors account for most of the myocardial infarction risk worldwide, with the only difference being the prevalence of some versus others depending on geographic risk. The Framingham Heart Study algorithm includes sex, age, blood pressure (treated and untreated), total and HDL cholesterol, diabetes and smoking habits (D'Agostino, R. B. et al. Circulation 117, 743-753 [2008]). It would also be reasonable to add obesity or waist circumference, physical inactivity and low consumption of fruit and vegetables, as suggested by the INTERHEART investigators (Yusuf, S. et al. Lancet 364, 937-952 [2004]). These predictive risk scores have been translated into guidelines and statements to allow individuals to adopt risk modification strategies on the basis of their CVD risk profile.

However, although such risk prediction algorithms can be of substantial value for population studies, their usefulness for primary care of the individual is questionable. More-simplistic approaches might be more likely to succeed in this setting, as the mathematical detail involved in the risk prediction algorithms might decrease their attractiveness to physicians. Indeed, when I write a scientific document or prepare a presentation on populations at risk, I find myself using the most sophisticated algorithms published. When I see an individual in search of his/her risk-factor profile, however, I pragmatically rely on the identification and modification of individual risk factors, and ignore formulas and algorithms. Although some of my colleagues would consider this approach to be 'scientific misbehavior', in December 2008, at the annual ACC cardiovascular symposium in New York, I asked an location, culture and ethnicity (Yusuf, S. et al. Lancet 364, 937-952 [2004]). Although they suggested that governments, national societies and foundations collaborate to target the overall risk-factor profile in the region-a process that might require conventional algorithms and risk factor scoring models - the INTERHEART investigators also suggested that simplistic surveillance management of each of the encountered risk factors should instead be used at the individual level.

The benefits of a simplistic approach to disease management in individuals can also be observed for management of individual risk factors. A good example is obesity, which seems to be the main underlying contributor to the evolving epidemic of CVD across the world and must, therefore, be prevented. Numerous diets, calorie counting and dietary formulas have been designed for the prevention and treatment of obesity but, after many trials of these strategic approaches, only one simple approach seems to be successful on a regular basis: eating less and exercising more.

A distinction must be made between population and individual targeting for the evaluation and management of the CVD risk factor profile. In order to succeed, primary prevention strategies for individual patients should be simple and personal.

doi:10.1038/nrcardio.2009.120
Valentin Fuster is the Editor-in-Chief of Nature Reviews Cardiology.

Competing interests The author declares no competing interests. 